Published in final edited form as:

Neurology. 2004 April 13; 62(7): 1120-1126.

\title{
LGI1 mutations in autosomal dominant partial epilepsy with auditory features
}

\author{
R. Ottman, PhD, M.R. Winawer, MD, MS, S. Kalachikov, PhD, C. Barker-Cummings, MPH, T.C. \\ Gilliam, PhD, T.A. Pedley, MD, and W.A. Hauser, MD \\ From the Gertrude H. Sergievsky Center (Drs. Ottman, Winawer, and Hauser, C. Barker- \\ Cummings), Department of Epidemiology (Drs. Ottman and Hauser, C. Barker-Cummings), \\ Mailman School of Public Health, Department of Neurology (Drs. Winawer, Pedley, and Hauser), \\ and Columbia Genome Center (Drs. Kalachikov and Gilliam), Columbia University, New York, NY; \\ and Epidemiology of Brain Disorders Department (Dr. Ottman), New York State Psychiatric Institute, \\ New York, NY.
}

\begin{abstract}
Objectives-Mutations in LGII cause autosomal dominant partial epilepsy with auditory features (ADPEAF), a form of familial temporal lobe epilepsy with auditory ictal manifestations. The authors aimed to determine what proportion of ADPEAF families carries a mutation, to estimate the penetrance of identified mutations, and to identify clinical features that distinguish families with and without mutations.
\end{abstract}

Methods-The authors sequenced LGII in 10 newly described ADPEAF families and analyzed clinical features in these families and others with mutations reported previously.

Results-Three of the families had missense mutations in LGII (C42R, I298T, and A110D). Penetrance was $54 \%$ in eight families with LGII mutations the authors have identified so far (five reported previously and three reported here). Excluding the original linkage family, the authors have found mutations in 50\% (7/14) of tested families. Families with and without mutations had similar clinical features, but those with mutations contained significantly more subjects with auditory symptoms and significantly fewer with autonomic symptoms. In families with mutations, the most common auditory symptom type was simple, unformed sounds (e.g., buzzing and ringing). In two of the newly identified families with mutations, some subjects with mutations had idiopathic generalized epilepsies.

Conclusions - LGII mutations are a common cause of autosomal dominant partial epilepsy with auditory features. Current data do not reveal a clinical feature that clearly predicts which families with autosomal dominant partial epilepsy with auditory features have a mutation. Some families with LGII mutations contain individuals with idiopathic generalized epilepsies. This could result from either an effect of $L G I I$ on risk for generalized epilepsy or an effect of co-occurring idiopathic generalized epilepsy-specific genes in these families.

Autosomal dominant partial epilepsy with auditory features (ADPEAF) is a form of idiopathic temporal lobe epilepsy with auditory ictal manifestations. ${ }^{1-4}$ In 1995 , we localized the gene to a 10-cM region on chromosome 10q22-24 in a single large family with autosomal dominant inheritance of epilepsy. ${ }^{1}$ Among 11 individuals with idiopathic epilepsy in the family, 55\% described auditory symptoms with their seizures. These included unformed sounds such as

Address correspondence and reprint requests to Dr. Ruth Ottman, G. H. Sergievsky Center, Columbia University, 630 West 168th Street, P\&S Box 16, New York, NY 10032; e-mail:ro6@columbia.edu.

Additional material related to this article can be found on the Neurology Web site. Go to www.neurology.org and scroll down the Table of Contents for the April 13 issue to find the title link for this article. 
buzzing, ringing, or clicking; distortions such as volume changes or muffling; and complex sounds such as a specific radio jingle or singer. ${ }^{3}$ One person described seizures precipitated by sounds.

In 1999, linkage was reported to an overlapping interval in a large Basque family with similar symptoms, which the authors called autosomal dominant lateral temporal lobe epilepsy. ${ }^{5}$ This report narrowed the minimal genetic region to approximately $3 \mathrm{cM}$, assuming the causative gene was the same in the two families. In 2002, positional cloning efforts focused on this 3cM interval led to the identification of the causative gene, leucine-rich glioma-inactivated 1 (LGII). ${ }^{6,7}$ Subsequently, a number of other mutations have been reported (figure 1). ${ }^{8-13}$

LGII is not homologous to any known ion channel, and the mechanism by which it causes epilepsy is unknown. Protein homology suggests it is likely to be involved in CNS development. ${ }^{6}$ The encoded protein, Lgi1, contains three leucine-rich repeats (LRRs) and aligns closely with other LRRs that bind nerve growth factor and other neurotrophins. ${ }^{6}$ $L G I 1$ is a member of a subfamily of LRR-encoding genes, denoted LGII, LGI2, LGI3, and LGI4. ${ }^{14}$

Expression of $L G I I$ is absent or significantly reduced in many high-grade, but not low-grade, gliomas, suggesting that it may play a role in progression of glial tumors. ${ }^{15-17}$ However, the gene is expressed primarily in neurons rather than glia, ${ }^{6,7}$ and no definite cases of glioblastoma have been reported in families with epilepsy caused by $L G I I$ mutations.

This article addresses three questions of importance with respect to LGII mutations: 1) how common are LGII mutations in families containing multiple individuals with ictal auditory symptoms; 2 ) what is the penetrance of $L G I 1$ mutations in families with ADPEAF; and 3) what is the range of phenotypic manifestations of mutations in LGII? Answers to these questions will help identify patients likely to carry mutations and may also provide clues to the mechanism by which mutations increase risk for epilepsy.

\section{Methods.}

\section{Clinical data collection.}

The current study included 10 newly tested families containing 2 or more subjects with idiopathic epilepsy with ictal auditory symptoms. Three of the 10 families were referred specifically because of auditory symptoms. The remaining 7 families were drawn from our database of 94 families collected for genetic linkage analysis. All families containing two or more subjects with ictal auditory symptoms were selected from the linkage database for inclusion. The linkage families were ascertained without regard to epilepsy syndrome or occurrence of auditory symptoms; hence, we also used them to estimate the proportion of familial focal epilepsy that meets criteria for ADPEAF.

Both sets of families were ascertained through a variety of mechanisms, including physician referrals from neurologists at our institution and elsewhere, self-referrals solicited through a study web site, flyers mailed to voluntary organizations, and presentations at meetings for people with epilepsy. The Columbia University Medical Center Institutional Review Board approved the study.

The protocol for clinical diagnosis and classification has been described in detail previously. 18 Briefly, we collected information on each family member using a set of validated semistructured interviews, usually administered by telephone. ${ }^{19,20}$ Whenever possible, we also collected medical records from the patients' treating physicians. These frequently contained additional seizure descriptions, histories of etiologic factors, and EEG and 
neuroimaging data. Some patients were also given a brief neurologic examination and a study EEG. We did not perform EEGs on unaffected individuals except when EEG data might have helped to confirm a suspected epilepsy diagnosis.

To derive a final diagnosis, the information collected on each subject was reviewed by experienced epileptologists (W.A.H. and T.A.P.), who were blinded to information about other family members. Interpretation of the data from medical records, especially EEG and neuroimaging reports, included an assessment of the quality of the information received. When an actual EEG tracing was included in the medical record, we reviewed it directly. When only an EEG report was available, we assessed its likely validity by determining whether it was performed at an established epilepsy center, the reporting electroencephalographer was board certified, and the description and clinical-electrographic correlation were consistent and appropriate. Reports of questionable validity were not used to make a diagnosis.

Epilepsy was defined as a lifetime history of two or more unprovoked seizures. Subjects with epilepsy whose first unprovoked seizure was preceded by an insult to the CNS were classified as remote symptomatic, and those with no identified cause were classified as idiopathic. Seizures precipitated by acute alterations in homeostasis or insults to the CNS (including febrile seizures) were excluded from the definition of epilepsy and classified as acute symptomatic. Seizures were classified according to the 1981 criteria of the International League Against Epilepsy (ILAE), ${ }^{21}$ and epilepsies were classified according to the 1989 ILAE criteria for classification of epilepsy syndromes. ${ }^{22}$

Because of our special interest in auditory symptoms, we added a question to the diagnostic interview to ask about them systematically, both preceding generalized tonic-clonic seizures and during small spells: "Do you usually hear any unusual sounds or have any change in your hearing? (If yes) What is the sound or change in your hearing?" In families in which two or more individuals with partial seizures responded "yes" to this question, we reviewed all of the information collected for each individual a second time to classify the semiology of partial seizures. We used five categories of semiology—sensory, motor, autonomic, psychic, and reflex - and subclassified sensory symptoms as auditory, visual, vertiginous, olfactory, gustatory, somatosensory, and cephalic. We also subclassified auditory symptoms into five categories: simple (e.g., buzzing, ringing, and humming), complex (e.g., voices or music), distortion (e.g., volume changes), cognitive (receptive aphasia), and reflex (seizures precipitated by sounds; see supplementary data, classification of auditory symptoms and tables E1 to E3, on the Neurology Web site for additional details; go to www.neurology.org).

\section{Molecular analysis.}

Whole blood samples were collected in sodium acid citrate dextrose tubes and processed within 3 days of collection. To detect sequence variants in $L G I 1$, we sequenced the gene's eight coding exons in DNA extracted from blood or EBV-transformed lymphoblastoid cell lines. PCR primers were placed at the exon boundaries to amplify complete exonic sequences and corresponding intron-exon junctions. PCR amplification products were purified over 96-well glass fiber plates (Whatman, Kent, UK) and sequenced in both directions using dye-terminator chemistry and ABI 373 automated sequencers (Applied Biosystems, Weiterstadt, Germany). Sequence variants were identified using the Sequencher3 program (Gene Codes Corporation, Ann Arbor, MI) and verified by manual inspection. Interpretation of the sequence analysis data was performed blind to disease status.

\section{Statistical analysis.}

All analyses were carried out with SPSS statistical software (Chicago, IL). We used Fisher's exact tests for categorical variables and t-tests for continuous variables. 


\section{Results.}

The 94 families collected for genetic linkage analysis contained 322 individuals with idiopathic epilepsy, of whom 169 had partial seizures. Thirty-four percent (58/169) of those with partial seizures had auditory symptoms. Fifty-one percent (48/94) of the families contained 2 or more subjects with idiopathic focal epilepsy, and of those, 19\% (9/48) met our criteria for ADPEAF (i.e., 2 or more subjects with ictal auditory symptoms). Two of the nine families had been tested previously and found to have mutations in LGII (Families 6610 and C). ${ }^{6}$ The remaining seven linkage families that met criteria for ADPEAF are reported here.

Given our known interest in this syndrome, we explored the possibility that subjects with auditory symptoms had been referred to us selectively, leading to an overestimate of the proportion of familial focal epilepsy meeting criteria for ADPEAF. For this purpose, we examined separately the subset of 31 families that had been ascertained in our original familial aggregation study, between 1985 and 1988, before publication of the article describing the syndrome in $1995 .{ }^{1}$ These 31 families were virtually identical to the remaining families, both in the proportion containing two or more subjects with idiopathic focal epilepsy (52\%) and in the proportion of those meeting criteria for ADPEAF (19\%). The proportion of individuals with partial seizures who had ictal auditory symptoms was slightly lower in these 31 families than in the others ( $27 \%$ vs $38 \%$ ), but the difference was not significant.

All 10 families included in the current study ( 7 from our linkage database and 3 others ascertained because of auditory symptoms) were of European descent. They contained 43 individuals with idiopathic epilepsy (4 primary generalized; 31 focal; 3 focal and primary generalized; and 5 unclassifiable). Seventy-nine percent (27/34) of those with partial seizures had ictal auditory symptoms. Age at onset averaged $14 \pm 1.95$ years (SE). None of the families contained an individual with glioblastoma.

We sequenced $L G I I$ in one affected subject from each family and identified mutations in three of them (two from the linkage database and one referred because of auditory symptoms). In the families of these three subjects, we sequenced the gene in all remaining sampled individuals and confirmed that the mutations cosegregated with epilepsy (figure 2).

Family $\mathrm{F}$ had a missense mutation in exon 1 (348T $>\mathrm{C}$, counting from the first nucleotide), resulting in a cysteine-toarginine substitution in amino acid residue 42 (C42R; see table and figure 2). Both affected sisters carried the mutation, as did their unaffected father.

Family G had a missense mutation in exon $8(1117 \mathrm{~T}>\mathrm{C})$, resulting in an isoleucine-to-threonine substitution in amino acid residue 298 (I298T). Five of the six affected individuals in the family had focal epilepsy with auditory symptoms. The remaining affected subject (IV:6) had two nocturnal generalized tonic-clonic seizures (GTCs) at age 10 and 11 years. No myoclonic or absence seizures were noted. An EEG report from the treating physician indicated generalized bursts of 2.5 to $3-\mathrm{Hz}$ spike- and slow-wave activity. She was treated successfully with valproic acid. We classified her syndrome as idiopathic generalized epilepsy (IGE), not otherwise specified (NOS), a category we used for IGE-like syndromes that do not fit clearly into existing IGE categories (e.g., because of atypical age at onset or seizure type constellations, or isolated GTCs that do not occur on awakening).

All four tested affected family members (including the one with IGE NOS) had the mutation. The remaining two affected subjects declined to give blood samples. Three unaffected individuals in the family (one of whom was married in) did not carry the mutation. Two other unaffected subjects did carry the mutation, consistent with reduced penetrance: II: 1 , aged 76 years, and IV:5, aged 17 years at the time of study. 
Family $\mathrm{H}$ had a missense mutation in exon $3(553 \mathrm{C}>\mathrm{A})$, resulting in an alanine-to-aspartate substitution in residue 110 (A110D). Three individuals had idiopathic epilepsy, and all had the mutation. Two were classified as having focal epilepsy with auditory symptoms, but both also had generalized-onset (myoclonic) seizures. II:2 had secondarily generalized seizures beginning at age 13 years, preceded by a warning of feeling light-headed and hazy, and complex partial seizures with similar symptoms, also including a high-pitched ringing in his right ear. $\mathrm{He}$ also had myoclonic seizures while watching television, beginning at age 21 years, and witnessed by his wife. An EEG tracing from his treating physician, which we reviewed directly, showed $3-\mathrm{Hz}$ generalized spike-wave activity during hyperventilation and photic stimulation. He was classified as having auditory partial epilepsy and juvenile myoclonic epilepsy.

Subject III: 1 had complex partial and secondarily generalized seizures preceded by auditory symptoms, and also myoclonic seizures. GTCs began at age 11 years and were preceded by a combination of auditory, visual, olfactory, and autonomic symptoms. In addition to these partial seizures, he had myoclonic seizures, beginning at age 15 years, with rapid jerks of the arms and legs while watching television and occurring most often after sleep deprivation. He also described occasional eyelid twitching with these episodes and had no alteration of consciousness or confusion during or after these events. An EEG report from his treating physician indicated sharp and slow-wave activity with right-sided predominance. He was also classified as having auditory partial epilepsy and juvenile myoclonic epilepsy.

Subject III:2, who was treated at our institution, had pyknolepsy, onset at age 3 years, with 4$\mathrm{Hz}$ generalized spike-wave and polyspike-wave activity on EEG. Absence seizures remitted at age 10 years. At age 22 years, she had two GTCs, the first $\sim 8$ months after a moderately severe head injury and the second $\sim 2$ months later. Both were nocturnal, with no warning or prodrome, and could not be classified further.

The positions of the three newly identified mutations within LGII and the alignments of the mutated Lgil proteins with other members of the $L G I$ gene family are shown in figure 1 . The C42R mutation in Family F affects the beginning of the cysteine-rich N-terminal LRR domain, which has cysteine in this position in all known Lgi proteins. The A110D mutation in Family $\mathrm{H}$ changes a highly conserved alanine to aspartate in the second LRR domain. The I298T mutation in Family G localizes to the protein's putative transmembrane domain and substitutes a polar amino acid (threonine) at a highly conserved position normally occupied by aliphatic amino acids isoleucine or valine.

We estimated penetrance in all eight families in which we have identified mutations so far (five reported previously and three reported here) ${ }^{6}$ We excluded the probands and their first-degree relatives from this analysis because their epilepsy histories had led to selection of the families for study. The analysis included 76 remaining family members (excluding married-in subjects) aged $>20$ years with unambiguous diagnoses ( 15 with idiopathic epilepsy and 61 unaffected). In this group of relatives, the proportion tested for mutations was $87 \%$ (13/15) in affected subjects but only $39 \%(24 / 61)$ in unaffected subjects. To correct for this bias, we estimated the number of carriers among the 37 unaffected subjects who were not tested. Among unaffected family members who were tested, $21 \%(5 / 24)$ were carriers. Assuming the same proportion applies to those who were not tested, $7.7(37 \times 0.21)$ would be expected to carry a mutation. Similarly, we assumed that both of the untested affected subjects had a mutation because all of the tested affected subjects did. Based on these calculations, we estimated that 27.7 relatives had a mutation (i.e., $5+15+7.7$ ), of whom 15 were affected, giving a penetrance estimate of $54 \%$ with an approximate $95 \%$ CI of 35 to $73 \%$.

After excluding the family used to define the syndrome initially, ${ }^{1}$ we compared the clinical features in the seven families with mutations and seven families without (see supplementary 
data on the Neurology Web site for additional details). The two sets of families were similar in the number of individuals with idiopathic epilepsy, age at onset, and distribution of seizure type. Subjects with partial seizures were more likely to have auditory symptoms in families with mutations than in those without ( $96 \%$ vs $72 \% ; p=0.049$; Fisher's exact test). Visual symptoms were less common in families with mutations than in families without ( $29 \%$ vs $48 \%$ ), but this difference was not significant. Autonomic symptoms were less common in families with mutations than in those without ( $17 \%$ vs $56 \% ; p=0.007$; Fisher's exact test). The two sets of families did not differ significantly in the types of auditory symptoms. In families with mutations, the most common type of auditory symptom was simple, unformed sounds such as buzzing and ringing (often accompanied by other auditory symptom types).

\section{Discussion.}

Mutations in LGII are a common cause of ADPEAF. Among 15 tested families, we have found mutations in 8 ( 5 reported earlier and 3 reported here). If we exclude the original family used to describe the syndrome and localize the gene, the proportion of tested families with mutations is $50 \%$ (7/14). The proportion of families with mutations will clearly depend on the criteria used to define ADPEAF. We have used a simple definition: two or more subjects with idiopathic focal epilepsy with ictal auditory symptoms. Our results suggest that families who meet this definition are likely to carry mutations in LGII.

In parallel with our findings, a European collaborative group has found LGII mutations in 5 of 10 families they have analyzed so far, assembled from Spain, Italy, and Germany. 7,13 They stated that families were selected based on autosomal dominant inheritance, seizures whose semiology indicated a lateral temporal lobe onset (not just auditory symptoms), absence of neurologic signs, and absence of known structural brain pathology. However, it is not straightforward to evaluate either consistency with autosomal dominant inheritance or consistency with lateral temporal lobe onset, and details of the specific criteria used were not given. Although auditory symptoms were not required for selection, all but one of their families had auditory symptoms; the remaining family had ictal aphasia without other auditory symptoms and was found to carry a mutation.

Our penetrance estimate of 54\% (95\% CI, 35 to $73 \%$ ) is lower than that reported previously $(71 \%) .{ }^{1}$ This lower estimate is probably explained, at least in part, by our correction for two biases that would have inflated the penetrance estimate. First, the families were ascertained because of multiple affected individuals, automatically inflating the proportion of tested individuals with idiopathic epilepsy. We corrected for this bias by excluding from the analysis probands and their first-degree relatives, whose epilepsy status defined eligibility of the families for inclusion. This approach reduced the number of relatives available for analysis and consequently also reduced the precision of our estimate. Second, affected family members were more likely to be tested for mutations than were unaffected family members (mostly because more unaffected subjects declined to give blood samples); therefore, a disproportionate number of unaffected mutation carriers were "missing" from the calculation. We corrected for this bias by estimating the number of untested family members likely to have a mutation. Although we corrected for these two biases, we could not correct for an additional selection bias inherent in the types of families we were studying. Given that these families contain a high proportion of affected individuals, they are automatically selected for high-penetrance mutations and for other genes or environmental factors that may influence expression. ${ }^{23} \mathrm{We}$ are currently working on other methods to evaluate penetrance that address potential biases and consider the age at onset of the disorder.

Clarification of the clinical features of epilepsy associated with LGII mutations is important to facilitate identification of families with mutations and to provide clues to the mechanism of 
action of the gene. However, current information does not point to a clinical feature that clearly predicts which families have mutations among those containing two or more subjects with auditory symptoms. The proportion of individuals with auditory symptoms is inflated in the families included here because they were selected to contain two or more such individuals. However, despite our use of the same criteria to ascertain all of the families, auditory symptoms were more common in families with mutations than in those without. With the present sample size, we did not detect any significant differences in the types of auditory symptoms in families with vs without. Additional investigation of auditory symptom types with a larger sample size is warranted.

The significantly lower prevalence of autonomic symptoms in families with mutations than in those without (16\% vs 56\%) may indicate less mesial temporal involvement. Autonomic symptoms, particularly epigastric or visceral sensations, are characteristic of mesial rather than lateral temporal lobe seizure onset, although they may occur because of spread from other brain regions.

The prevalence of auditory symptoms in the families in our linkage study was surprisingly high. Overall, $34 \%$ of subjects with partial seizures in these families reported auditory symptoms, and among families containing two or more subjects with focal epilepsy, $19 \%$ met our criteria for ADPEAF. Selective referral of families with these symptoms because of our known interest in ADPEAF is not a likely explanation. Families ascertained before recognition of the syndrome were just as likely to contain two or more subjects with auditory symptoms as were those ascertained later. The proportion of individuals with partial seizures who had auditory symptoms was somewhat lower in families ascertained before syndrome recognition than in those ascertained later ( $27 \%$ vs $38 \%$ ), but the difference was not significant. Also, in the families ascertained before symptom recognition, some of the data were collected before we added a specific question about auditory symptoms to the interview. The lower proportion of subjects with auditory symptoms in these earlier families may indicate that auditory symptoms are likely to be missed unless a specific question is asked, which is rarely done in clinical practice.

Our linkage families were not sampled systematically from a known population; therefore, the frequency of auditory symptoms, or of ADPEAF, remains to be determined in an unselected sample of epilepsy patients or families containing multiple affected persons. In any given collection of families, the proportion with ADPEAF depends on the proportion containing two or more subjects with idiopathic focal epilepsy (because this is a minimum requirement for ADPEAF). Further, among families containing two or more individuals with idiopathic focal epilepsy, the proportion with ADPEAF probably increases with increasing number of affected individuals. In our linkage database, the proportion of families meeting criteria for ADPEAF was 13\% (5/39) in families containing 2 or 3 individuals with idiopathic focal epilepsy, but $44 \%$ (4/9) in families containing 4 or more individuals with idiopathic focal epilepsy.

The three new missense mutations reported here are all likely to be pathogenic. None was found in a set of 123 control subjects ( 246 chromosomes) of European ancestry we studied previously; all segregated with disease in the families in which they were found, and all affect conserved amino acid residues. ${ }^{6}$ The mutations in Families $\mathrm{F}$ and $\mathrm{H}$ are in the extra-cellular portion of the protein and probably change the properties of the block of LRR domains known to be involved in protein-protein interactions. ${ }^{24}$ The mutation in Family $\mathrm{G}$ is the first found to localize to Lgil's putative transmembrane region (see figure 1). Functional studies of the effects of all three mutations on neuronal excitability would be of great interest but are not possible at this time because of limited understanding of the gene's effect. 
The mutation in Family F, C42R, is only four amino acids away from the C46R mutation identified in two families, one Norwegian 8 and the other Italian. ${ }^{9,10}$ Haplotype analysis of the two families with the C46R mutation is needed to explore their ancestral relationship; if they are truly unrelated, the finding of two closely located mutations in three families may indicate a mutation hotspot. Both families with the C46R mutation had sensory aphasia as an important part of the phenotype. ${ }^{9,25}$ In Family F, both affected individuals also reported difficulty in understanding language at the onset of their seizures. The occurrence of aphasia in all three families raises the possibility that these mutations have similar functional effects. However, aphasia has also been found in two families with mutations in exon 8: Family G, in which three of the five subjects with focal epilepsy had aphasia, and another Italian family reported recently with the $1519 \mathrm{~T}>\mathrm{A}$ mutation. ${ }^{13}$

We have found no definite cases of glioblastoma in the families with mutations, although one person in the original linkage family died of a brain tumor of unspecified type. ${ }^{6}$ This does not prove risk is not increased in these families because glioblastoma is rare, and few cases would be expected with such a small sample size. However, it does argue against a dramatic increase in risk.

Finally, one of our most interesting findings is the occurrence of IGEs in four subjects with mutations in two families with LGII mutations. In Family G, the subject with IGE NOS had only two seizures, both while asleep, and was classified as having generalized epilepsy based on her EEG. Her epilepsy may have been focal, and the generalized epilepti-form EEG abnormality may have been coincidental, or the original EEG may have been misinterpreted (although the EEG report appeared likely to be valid). In Family H, two subjects (II:2 and III: 1) had generalized (myoclonic) seizures and focal epilepsy with auditory symptoms, and the other (III:2) had pyknolepsy followed by unclassifiable GTCs after a head injury of uncertain significance. The constellation of clinical features in Family $\mathrm{H}$ may be caused by two genotypes (LGII and an unidentified IGE genotype) segregating within the same family, each of which increases risk for a different type of epilepsy. Alternatively, LGII mutations may increase risk for generalized and focal epilepsy, with phenotypic expression determined by other interacting genes or environmental factors. Molecular investigation of $L G I l$ 's function is needed to clarify how mutations increase risk for epilepsy and what phenotypes they are likely to produce. Analysis of the phenotypes associated with mutations, such as that reported here, is essential to interpret the functional data and may provide clues to function before molecular data are available.

\section{Supplementary Material}

Refer to Web version on PubMed Central for supplementary material.

\section{Acknowledgements}

The authors thank the families for their participation in this research. They also thank Wanda Garcia, Walkiria Jimenez, MS, and Carmen Liriano, MD, for their assistance with data collection.

\section{References}

1. Ottman R, Risch N, Hauser WA, et al. Localization of a gene for partial epilepsy to chromosome 10q [see comments]. Nat Genet 1995;10:56-60. [PubMed: 7647791]

2. Ottman R, Barker-Cummings C, Lee JH, Ranta S. Genetics of autosomal dominant partial epilepsy with auditory features. In: Berkovic SF, Genton P, Hirsch E, Picard F, eds. Genetics of Focal Epilepsies: Clinical Aspects and Molecular Biology. London: John Libbey, 1999:95-102.

3. Winawer MR, Ottman R, Hauser WA, Pedley TA. Autosomal dominant partial epilepsy with auditory features: defining the phenotype. Neurology 2000;54:2173-2176. [PubMed: 10851389] 
4. Winawer MR, Martinelli Boneschi F, Barker-Cummings C, et al. Four new families with autosomal dominant partial epilepsy with auditory features: clinical description and linkage to chromosome 10q24. Epilepsia 2002;43:60-67. [PubMed: 11879388]

5. Poza JJ, Saenz A, Martinez-Gil A, et al. Autosomal dominant lateral temporal epilepsy: clinical and genetic study of a large Basque pedigree linked to chromosome 10q. Ann Neurol 1999;45:182-188. [PubMed: 9989620]

6. Kalachikov S, Evgrafov O, Ross B, et al. Mutations in LGII cause autosomal-dominant partial epilepsy with auditory features. Nat Genet 2002;30:335-341. [PubMed: 11810107]

7. Morante-Redolat JM, Gorostidi-Pagola A, Piquer-Sirerol S, et al. Mutations in the LGII/Epitempin gene on 10q24 cause autosomal dominant lateral temporal epilepsy. Hum Mol Genet 2002;11:11191128. [PubMed: 11978770]

8. Gu W, Brodtkorb E, Steinlein OK. LGI1 is mutated in familial temporal lobe epilepsy characterized by aphasic seizures. Ann Neurol 2002;52:364-367. [PubMed: 12205652]

9. Pizzuti A, Flex E, Di Bonaventura C, et al. Epilepsy with auditory features: a LGI1 gene mutation suggests a loss-of-function mechanism. Ann Neurol 2003;53:396-399. [PubMed: 12601709]

10. Pizzuti A, Giallonardo AT. Correction. Ann Neurol 2003;54:137.

11. Fertig E, Lincoln A, Martinuzzi A, Mattson RH, Hisama FM. Novel LGI1 mutation in a family with autosomal dominant partial epilepsy with auditory features. Neurology 2003;60:1687-1690. [PubMed: 12771268]

12. Wallace RH, Izzillo P, Macintosh AM, Mulley JC, Berkovic SF. Mutations in LGII in an Australian family with familial temporal lobe epilepsy with auditory features [abstract]. Am J Hum Genet 2002;71:s472.

13. Michelucci R, Poza JJ, Sofia V, et al. Autosomal dominant lateral temporal epilepsy: clinical spectrum, new epitempin mutations, and genetic heterogeneity in seven European families. Epilepsia 2003;44:1289-1297. [PubMed: 14510822]

14. Gu W, Wevers A, Schroder H, et al. The LGII gene involved in lateral temporal lobe epilepsy belongs to a new subfamily of leucine-rich repeat proteins. FEBS Lett 2002;519:71-76. [PubMed: 12023020]

15. Chernova OB, Somerville RP, Cowell JK. A novel gene, LGI1, from 10q24 is rearranged and downregulated in malignant brain tumors. Oncogene 1998;17:2873-2881. [PubMed: 9879993]

16. Somerville RP, Chernova O, Liu S, Shoshan Y, Cowell JK. Identification of the promoter, genomic structure, and mouse ortholog of LGI1. Mamm Genome 2000;11:622-627. [PubMed: 10920229]

17. Cowell JK. Epilepsy research gets new guidance. Nat Med 2002;8:219-220. [PubMed: 11875488]

18. Winawer MR, Rabinowitz D, Barker-Cummings C, et al. Evidence for distinct genetic influences on generalized and localization-related epilepsy. Epilepsia 2003;44:1176-1182. [PubMed: 12919389]

19. Ottman R, Hauser WA, Stallone L. Semistructured interview for seizure classification: agreement with physicians' diagnoses. Epilepsia 1990;31:110-115. [PubMed: 2406127]

20. Ottman R, Lee JH, Hauser WA, et al. Reliability of seizure classification using a semistructured interview. Neurology 1993;43:2526-2530. [PubMed: 8255451]

21. Commission on Classification and Terminology ILAE. Proposal for revised clinical and electroencephalographic classification of epileptic seizures. Epilepsia 1981;22:489-501. [PubMed: 6790275]

22. Commission on Classification and Terminology ILAE. Proposal for revised classification of epilepsies and epileptic syndromes. Epilepsia 1989;30:389-399. [PubMed: 2502382]

23. Begg CB. On the use of familial aggregation in population-based case probands for calculating penetrance. J Natl Cancer Inst 2002;94:1221-1226. [PubMed: 12189225]

24. Scheel H, Tomiuk S, Hofmann K. A common protein interaction domain links two recently identified epilepsy genes. Hum Mol Genet 2002;11:1757-1762. [PubMed: 12095917]

25. Brodtkorb E, Gu W, Nakken KO, Fischer C, Steinlein OK. Familial temporal lobe epilepsy with aphasic seizures and linkage to chromosome 10q22-q24. Epilepsia 2002;43:228-235. [PubMed: 11906506] 
A

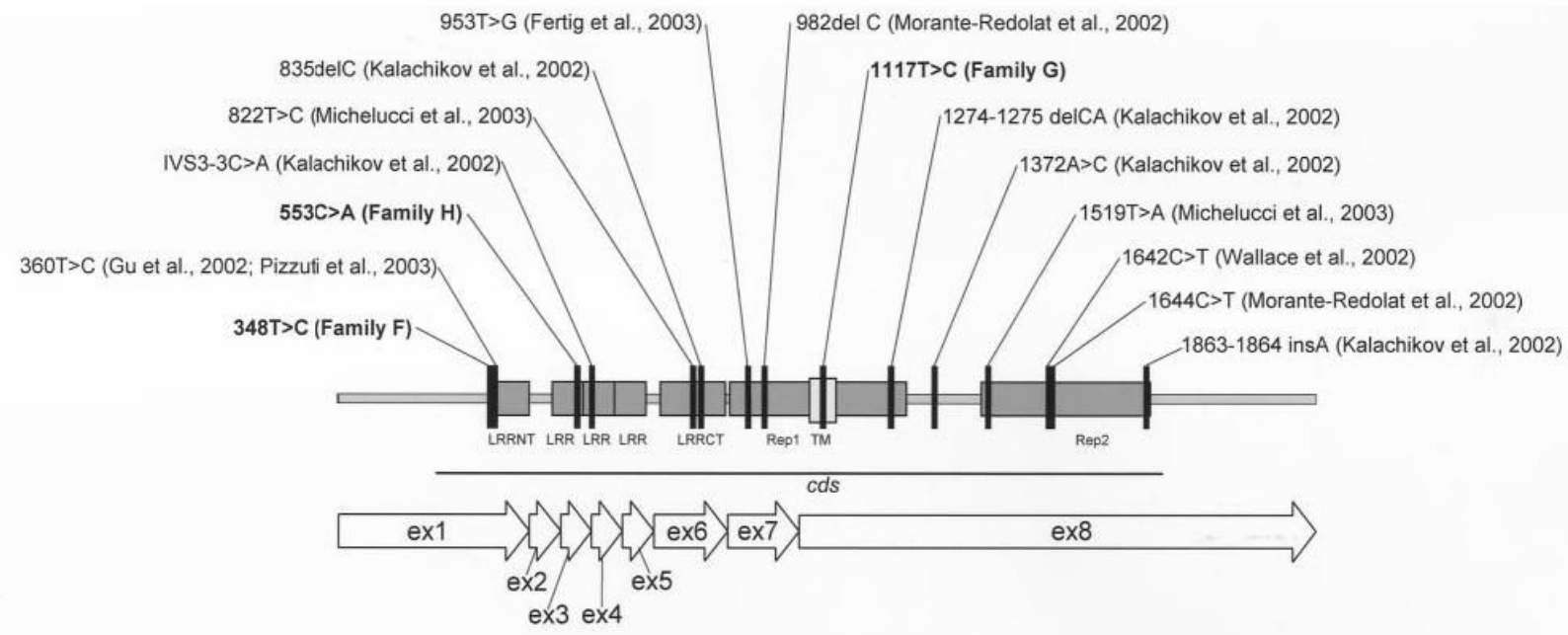

B

LGI1 mRNA (NM_005097), 2254 bp

FAMILY $\mathbf{F}$

hLgI1 PAKPKCPAVCTCTKDNALCENARSIPRTV

hLgi2 RRLARCPATCSCTKESIICVGSSWVPRIV

hLqi3 PKTPPCPPSCSCTRDTAFCVDSKAVPRNI

hLgi4 PPKGKCPLRCSCSKDSALCEGSPDLPVSE

mLgiI PAKPKCEAVCTCSKDNALCENARSIPRTV

mLgi2 RRLARCPATCSCTKESIICVGSSWVRIV

mLqi4 PPKGKCPPHCSCSKENTLCEGSPELPESE

mLgi3 PPKGKCPPHCSCSKENTLCEGSPELPESE

rLgi1 PAKPKCPAVCTCSKDNALCENARSIPRTV

Family $F$
FAMILY $G$

hLgil CKPIVIETQLYVIVAQLFGGSHIYKRDSE

hLgi2 CKAILIDDQVFVVVAQLFGGSHIYKYDES

hLgi3 CKPMVVDSOLYVVVAOLFGGSYIYHWDPN

hLgi4 CKPLVLGPSLFVLAARLWGGSOLWARPSP

mLgi1 CKDIVIDTQLYVIVAQLFGGSHIYKRDGE

mLgi2 CKAILIDDQVEVVVAQLFGGSHIYKYDES

mLqi4 CKPLVLGPRLFILAARLWGGSOLWSRSSE

mLgi3 CKPLVLGPRLFILAARLWGGSOLWSRSSP

rLgi1 CKPIVIDTQLYVIVAQLFGGSHIYKRDGE

Family G
FAMILY H

hLgi1 SNSEDVISDDAFIGLPHLEYLFIENNNI

hLgi2 SNSETIIRDDAEAGLFHLEYLFIEGNKI

hLgI 3 SNKETLIGDNAETGLSHLOYLEIENNDI

hLgi4 SNSESVIEDDAFAGLSHLQYLFIEDNEI

mLgII SNSEDVISDDAEIGLFHLEYLFIENNNI

mLgi2 SNSETVIRDDAEAGLFHLEYLFIEGNKI

mLgi4 SNTFSVIEGDAFIGLSYLQYLFIEDNKI

mLgI3 SNTESVIEGDAFIGLSYLQYLEIEDNKI

rLgiI SNSEDVISDDAFIGLPHLEYLFIENNNI CKPIVIETQLYVTVAOLFGGSHIYKRDSF Eamily $\mathrm{H}$

1298T $\frac{\text { SNSEDVISDDAFIGLPHLEYLFIENNNI }}{\text { SNSEDVISDDDFIGLPHLEYLFIENNNI }}$

Figure 1.

(A) Positions of LGII mutations in published families with autosomal dominant partial epilepsy with auditory features, counted from the first nucleotide of the LGII mRNA sequence from GenBank accession no. NM_005097. In the reports of the European collaborative group, ${ }^{\text {, }}$ 13 the mutations were counted from the initiation codon; these have been renumbered from the first nucleotide. In addition, the $1320 \mathrm{C}>\mathrm{T}$ mutation was corrected to $1420 \mathrm{C}>\mathrm{T}$ (counting from the initiation codon; J. Perez-Tur, personal communication); this is shown as $1644 \mathrm{C}>\mathrm{T}$ counting from the first nucleotide. (B) Positions of the amino acid substitutions on the multiple alignments of Lgi proteins in human, mouse, and rat. 
Family F: $348 \mathrm{~T}>\mathrm{C}$ (exon 1) $\mathrm{C} 42 \mathrm{R}$

Family G:

$1117 \mathrm{~T}>\mathrm{C}$ (exon 8) I298T

\section{Family $\mathrm{H}$ : $553 C>A($ exon 3$)$ $\mathrm{A} 110 \mathrm{D}$}
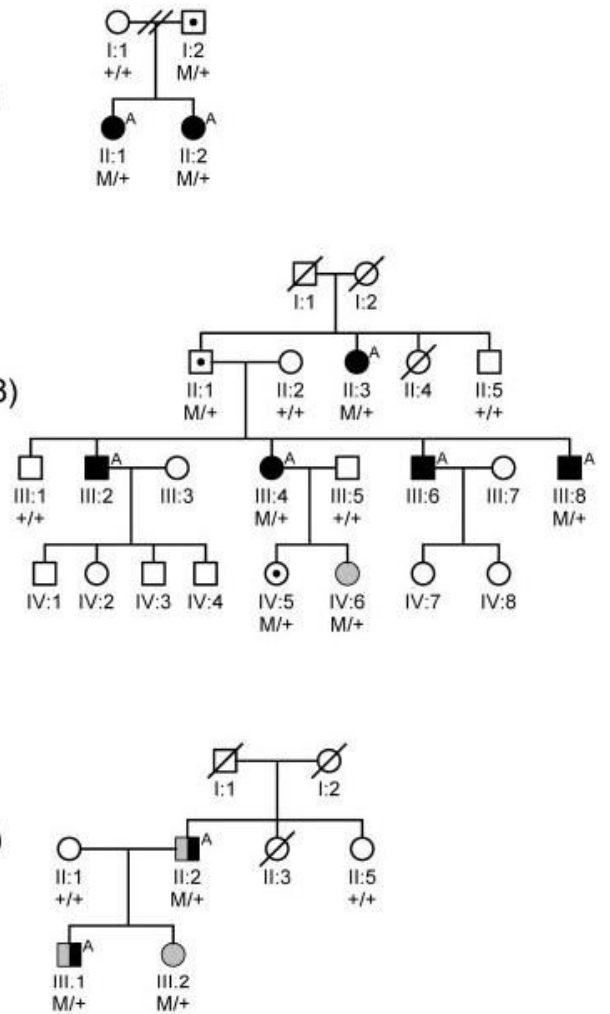

Figure 2.

Families with newly identified mutations in LGI1. M/+indicates mutation carrier; +/+ indicates no mutation. Symbol definitions: $\square \circ=$ Unaffected; $\boldsymbol{\bullet}^{\mathrm{A}} \bullet \mathbf{A}=$ Idiopathic partial epilepsy with auditory features; $\square \cdot=$ = Unaffected mutation carrier; $\mathbb{W} \mathbb{D}=$ = Idiopathic generalized epilepsy; प $\mathbf{O}$ = Idiopathic partial and generalized epilepsy. 


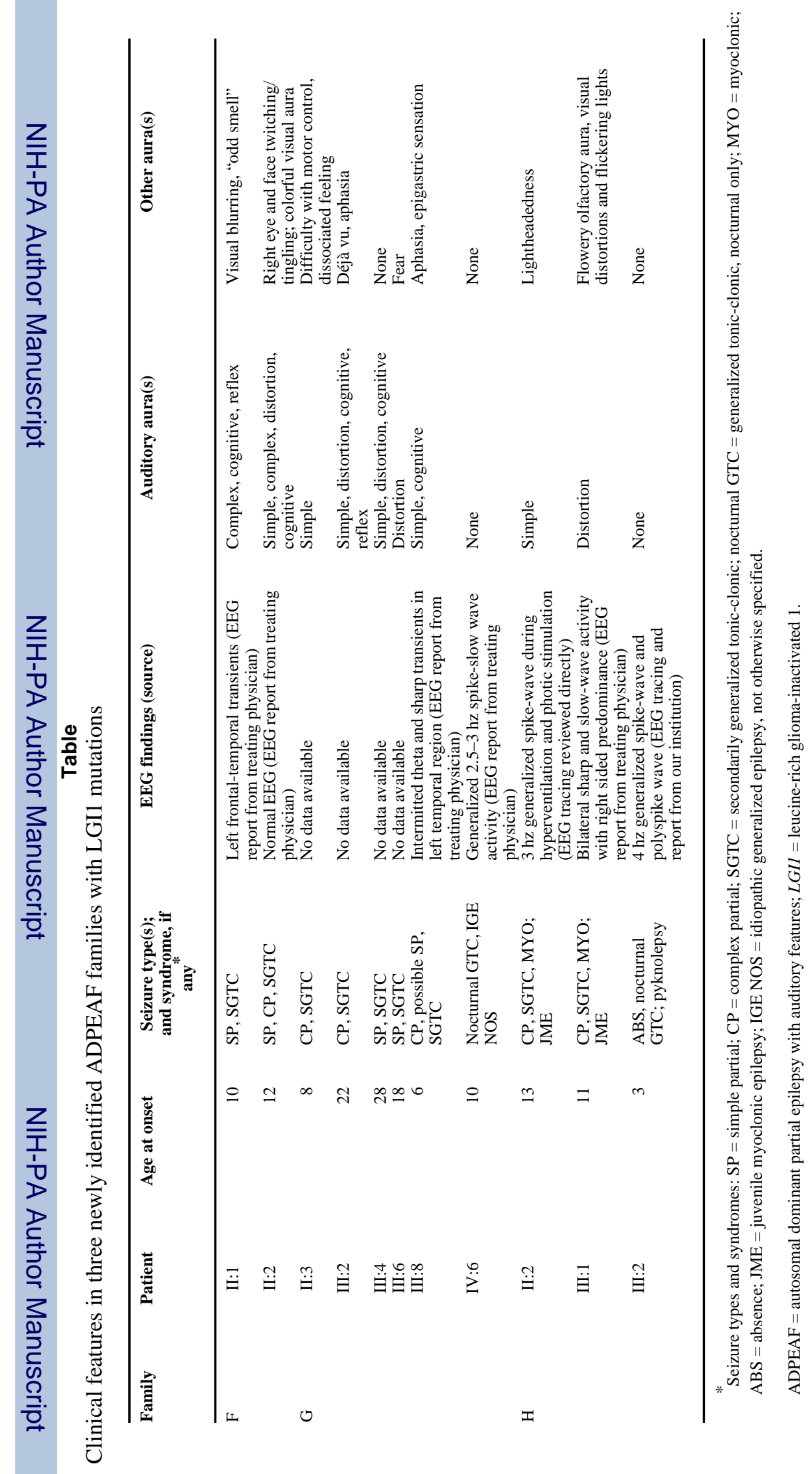

